\title{
Resistance of Young Rat Hepatic Mitochondria to Bile Acid-Induced Permeability Transition: Potential Role of $\alpha$-Tocopherol
}

\author{
ERIC GUMPRICHT, MICHAEL W. DEVEREAUX, ROLF DAHL, JASON S. SODEN, GENEVIEVE C. SPARAGNA, \\ SCOTT W. LEONARD, MARET G. TRABER, AND RONALD J. SOKOL
}

\begin{abstract}
Department of Pediatrics [E.G., M.W.D., R.D., J.S.S., R.J.S.], University of Colorado Denver School of Medicine, Aurora, Colorado 80045; Department of Integrative Physiology [G.C.S.], University of Colorado at Boulder, Boulder, Colorado 80309; Linus Pauling Institute [S.W.L., M.G.T.], Oregon State University, Corvallis, Oregon 97331
\end{abstract}

\begin{abstract}
Retention of bile acids within the liver is a primary factor in the pathogenesis of cholestatic liver disorders, which are more common in human infants. The objective of this study was to evaluate developmental changes in mitochondrial factors involved in bile acid-induced hepatocyte injury. Hepatic mitochondria from adult rats (aged 9 wk) underwent a mitochondrial permeability transition (MPT) and release of cytochrome $c$ upon exposure to glycochenodeoxycholic acid. In contrast, mitochondria from young rats (age 6-36 d) were resistant to MPT induction and cytochrome $c$ release. Neither mitochondrial levels of MPT-associated proteins (voltage-dependent anion channel, cyclophilin D, or adenine nucleotide translocase), Bcl-2 family proteins, nor antioxidant enzymes explained this resistance. Mitochondria from young rats contained 2- to 3-fold higher $\alpha$-tocopherol ( $\alpha$-TH). In vivo $\alpha$-TH enrichment of adult hepatic mitochondria increased their MPT resistance. Tetra-linoleoyl cardiolipin (TL-CL), the primary molecular species of CL, was reduced in mitochondria of the young rat; however, enrichment with CL and TL-CL only modestly increased their MPT susceptibility. In conclusion, we observed an unexpected resistance in young rats to bile acid induction of mitochondrial cell death pathways, which may be related to developmental differences in membrane composition. (Pediatr Res 64: 498-504, 2008)
\end{abstract}

$\mathrm{D}$ espite recent advances in elucidating the mechanisms of liver injury in cholestatic disorders, the development of effective therapies has been hampered by a lack of potential therapeutic targets. For these reasons, cholestatic diseases are among the leading indications for liver transplantation. The human neonate is exceptionally prone to the development of cholestasis (1). Characteristic features of neonatal cholestasis

Received April 16, 2008; accepted June 10, 2008

Correspondence: Ronald J. Sokol, M.D., Professor and Vice Chair of Pediatrics, Department of Pediatrics, University of Colorado Denver School of Medicine, The Children's Hospital, 13123 E. 16th Avenue Box B290, Aurora, CO 80045; e-mail: sokol.ronald@tchden.org

Supported in part by grants to RJS from the NIH (RO1DK038446); The Children's Hospital Research Institute and the Abby Bennet Liver Research Fund, and grants to MGT from the NIH (RO1DK59576) and the Environmental Health Sciences Center at Oregon State University (NIEHS P30 ES00210).

Presented in part at the 56th Annual Meeting of the American Association for the Study of Liver Diseases, San Francisco, CA, November 2005, and published in abstract form (Hepatology 2005; 777A); and at the 58th Annual Meeting of the American Association for the Study of Liver Diseases, Boston, MA, November 2007 and published in abstract form (Hepatology 2007: 516 A).

Supplemental materials, which accompany this article, are available online at www. pedresearch.org include histologic evidence of hepatocyte swelling (2) and elevated circulating hepatocellular enzymes (aspartate aminotransferase (AST) and alanine aminotransferase (ALT)), both of which suggest oncotic necrosis in the pathogenesis of cell injury. It is not well understood why the neonatal hepatocyte has this increased susceptibility to cholestatic injury, although, proposed factors include immaturity of synthesis, hepatocyte uptake, and canalicular transport of bile acids (1), reduced antioxidant defense systems $(3,4)$, or other developmental factors.

The accumulation of hydrophobic bile acids in hepatocytes, such as glycochenodeoxycholic acid (GCDC), is a primary factor in the pathogenesis of cholestatic liver injury. Although the precise mechanisms contributing to bile acid-induced toxicity are unknown, several lines of evidence suggest that these compounds directly target hepatocyte mitochondria perturbing mitochondrial homeostasis, culminating in apoptotic and necrotic cell death. For example, liver mitochondria exposed to hydrophobic bile acids have reduced electron transport and impaired mitochondrial respiration $(5,6)$, findings which have also been demonstrated in the bile duct-ligated model of cholestatic injury (7). Hepatocytes exposed to bile acids demonstrate changes in mitochondrial function (induction of mitochondrial permeability transition, MPT) that trigger cell death (8). The MPT is characterized by mitochondrial swelling and outer membrane permeabilization, followed by cytochrome $c$ release, a pivotal event during apoptotic cell death. Furthermore, several laboratories have implicated oxidative stress as a primary factor in bile acid-induced MPT $(9,10)$, which is ameliorated with antioxidants (9). In addition to direct effects on mitochondria, bile acids can also promote necrotic or apoptotic cell death through activation and/or translocation of critical cell signaling proteins. Bile acid effects on death receptors (Fas, TRAIL) facilitate the translocation of the pro-apoptotic Bcl-2 regulatory protein Bax from cytosol to mitochondria, wherein the protein inserts itself into the outer membrane inducing the MPT and activating down-

\footnotetext{
Abbreviations: $\boldsymbol{\alpha}$-TH, alpha-tocopherol; $\boldsymbol{\alpha}$-TTP, alpha-tocopherol transfer protein; CL, cardiolipin; GSH-Px, glutathione peroxidase; GSSG-R, glutathione reductase; GCDC, glycochenodeoxycholic acid; MPT, mitochondrial permeability transition; ROS, reactive oxygen species; TL-CL, tetra-linoleoyl CL
} 
stream caspases (11). Although bile acid-mediated toxicity acts, in part, through death receptor-mediated hepatocyte cell death (12), this signaling pathway alone does not account for the full extent of cholestatic injury (13).

The objective of this study was to evaluate developmental effects on the function and composition of hepatic mitochondria in relation to bile acid induction of mitochondrial pathways of cell death. Isolated hepatic mitochondria were used as the experimental model in this study in preference to cultured hepatocytes for several reasons. First, culturing primary hepatocytes from suckling rats poses experimental problems including: a) technical difficulties in isolating sufficient quantities of hepatocytes from suckling rats for use as the experimental model, and b) the developmentally regulated marked reduction in bile acid uptake by suckling rat hepatocytes (14). Thus, inducing toxicity by incubating hepatocytes with bile acids, the standard experimental model, would not be possible because of severely impaired bile acid uptake by neonatal hepatocytes. Second, mitochondrial cell death pathways can be directly evaluated in purified mitochondria, which allow for in vitro manipulation of membrane composition that can be used to delineate the effects of membrane components. In this study, isolated mitochondria from young, suckling rats exhibited marked resistance to bile acid-induced MPT, which may be related to mitochondrial membrane composition.

\section{MATERIALS AND METHODS}

Materials. (See Supplemental Materials online at www.pedresearch.org). Isolation of rat liver mitochondria. Rat liver mitochondria were isolated from adult and young rats by differential centrifugation (Supplemental Materials online at www.pedresearch.org). All animals received humane care in compliance with the Committee on Laboratory Animal Research of the University of Colorado Denver Health Sciences Center.

MPT measurements. The MPT was determined spectrophotometrically at $540 \mathrm{~nm}$ (Supplemental Materials online).

Glutathione, glutathione-dependent enzyme activity and vitamin E levels. Mitochondrial glutathione $(\mathrm{GSH})$ concentrations were determined using a commercial spectrophotometric-based kit (QuantiChrom Glutathione Assay Kit: DIGT-250, Bioassay Systems, Hayward, CA). Glutathione peroxidase (GSH-Px) activity and glutathione reductase (GSSG-R) were determined according to the methods of Lawrence and Burk (15), and Carlberg and Mannervik (16), respectively, and expressed as units/mg pro. $\alpha$-TH and $\gamma$-tocopherol $(\gamma$-TH)) was determined in liver homogenate and mitochondrial fractions by HPLC with electrochemical detection $(17,18)$.

Measurement of ROS generation. Generation of reactive oxygen species (ROS) was determined by dichlorofluorescin (DCF) fluorescence, as previously described (19).

SDS-PAGE and immunoblotting. Proteins were subject to SDS-PAGE and immunoblotting as previously described (Supplemental Materials online).

Enrichment of liver mitochondrial $\alpha-T H$ in adult rats. Adult rats were treated with s.c. injections of Vital E-300 (20). Vital E-300 is a veterinary injectable form of $\mathrm{d}-\alpha$-TH containing $300 \mathrm{IU} / \mathrm{mL}$ of $\mathrm{d}-\alpha-\mathrm{TH}$ compounded with $20 \%$ ethanol, $1 \%$ benzyl alcohol in an emulsifiable base. Adult rats were administered a single s.c. injection of Vital E-300 $(2.5,5.0$, or $10 \mathrm{mg} / 100 \mathrm{~g}$ body weight) or saline to achieve a range of anticipated $\alpha$-TH levels in hepatic mitochondria. After $48 \mathrm{~h}$, mitochondria were subject to GCDC-induced MPT and $\alpha$-TH determination.

Isolation and analysis of cardiolipin and cholesterol. Total and individual cardiolipin (CL) molecular species were quantified from rat liver mitochondria by electrospray ionization mass spectrometry (21). The quantities of individual CL species were calculated based upon the relative ratios of peak areas of the specific molecular species to the area of the internal standard and expressed as nmoles of individual fatty acyl side chain CL species (carbon number:number of double bonds)/mg protein, using a standard curve.

Mitochondrial cholesterol was measured fluorimetrically using an Amplex Red assay kit (Invitrogen/Molecular Probes, Eugene, OR).
Enrichment of cardiolipin into young rat liver mitochondria. To enrich mitochondria from livers of young rats, the amount of added CL required to achieve levels in the range of that measured in the adult was calculated. To compensate for the poor permeability of phospholipids into mitochondrial membranes (22), a 10 -fold $(11.7 \mathrm{nmol} / \mathrm{mL}$ mitochondria) and 100 -fold (117 $\mathrm{nmol} / \mathrm{mL}$ mitochondria) excess was used. The appropriate amount of bovine liver CL, containing approximately 50\% tetra-linoleoyl cardiolipin (TL-CL), dissolved in ethanol, or ethanol alone (solvent control), was added to flasks and the solvent removed by evaporation under a $\mathrm{N}_{2}$ stream. To the dried lipid, $10 \mathrm{~mL}$ hepatic mitochondria suspended in MPT buffer (approx. $1 \mathrm{mg}$ pro/mL) was added and the samples were incubated for $30 \mathrm{~min}$ at $28^{\circ} \mathrm{C}$. After the incubation period, the mitochondria were centrifuged at $10,000 \mathrm{~g}$ for $10 \mathrm{~min}$ and the enriched mitochondrial pellets were resuspended to the original volume and subjected to the MPT assay. Aliquots were also stored at $-70^{\circ} \mathrm{C}$ for analysis of total and individual CL subspecies.

Statistical analysis. Statistical comparisons among experimental groups were conducted by either an ANOVA or student $t$ test. For analyzing the association between mitochondrial $\alpha$-TH levels and mitochondrial swelling, a least squared regression curve was calculated. A $p$-value of $<0.05$ was considered statistically significant.

\section{RESULTS}

Rat liver mitochondria exhibit age-related resistance to GCDC-induced MPT. Mitochondria from young rat livers exposed to $100 \mu \mathrm{M}$ GCDC demonstrated significant resistance to MPT induction compared with adult rats (representative MPT tracing shown in Fig. 1A). This age-related resistance to GCDC-induced mitochondrial swelling was also concentration-dependent (Fig. 1B). Resistance to GCDCinduced swelling in young rat mitochondria was also confirmed morphologically by electron microscopy (Fig. 1C). In addition, mitochondria from young rats maintained their cytochrome $c$ content when exposed to 25-100 $\mu \mathrm{M}$ GCDC, whereas adult liver mitochondria released cytochrome $c$ at 100 $\mu \mathrm{M}$ GCDC (Fig. 2). Cyclosporin A (CsA) prevented GCDCinduced swelling in mitochondria and cytochrome $c$ release at all ages (data not shown), indicating that these effects were caused specifically by the MPT.

To investigate the factors causing MPT resistance in young rats, in subsequent experiments we compared mitochondria from 10-day-old rats (representative of the young rats) with those of adult rats.

Mitochondria from young rats are resistant to other MPT inducers. In these experiments, adult mitochondria incubated with $100 \mu \mathrm{M}$ GCDC underwent an MPT that was 4-fold greater than that of the 10-days old (Fig. 3). Adult mitochondria incubated with the thiol-modifying reagent phenylarsine oxide (PhASO), or the peroxides, $t$-butyl hydroperoxide $(t-\mathrm{BOOH})$ and hydrogen peroxide $\left(\mathrm{H}_{2} \mathrm{O}_{2}\right)$, also underwent significant swelling, which were inhibited by CsA (data not shown). Mitochondria from 10-day-old rats underwent similar swelling with PhASO, as did the mitochondria from adult rats; however, the 10-day-old rat mitochondria responded with $<50 \%$ of the adult response to the peroxides. Thus, the resistance of the 10-day-old rat to undergo an MPT was greatest with GCDC, substantial with peroxides, and absent with PhASO.

Mitochondrial redox status and protein expression in adult and young rat liver. ROS generation in the presence of GCDC was similar in liver mitochondria from 10-day-old and adult rats (Fig. 4A). Despite greater GSH-Px and GSSG-R activities in adult rats (Fig. 4B), GSH concentrations were similar in young and adult mitochondria, suggesting that 

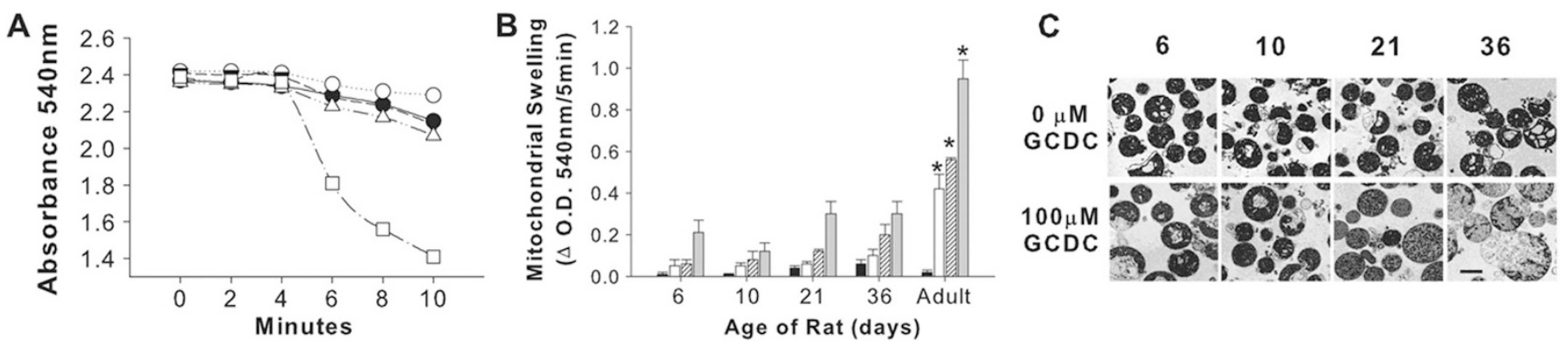

Figure 1. (A) A representative tracing demonstrating age-related resistance to GCDC-induced MPT in 6-days old $(\bullet)$, 10-days old $(\bigcirc)$, 21-days old $(\mathbf{\Delta})$, 36-days old $(\triangle)$, and adult $(\square)$ rat mitochondria exposed to $100 \mu \mathrm{M} \mathrm{GCDC}$ (added at $5 \mathrm{~min}$ ). Note that $y$ axis values do not begin at "0". (B) Mean values \pm SEM of rats of different ages during the time course of MPT induction by $0 \mu \mathrm{M}(\square), 25 \mu \mathrm{M}(\square), 50 \mu \mathrm{M}(\square)$ and $100 \mu \mathrm{M}$ GCDC ( $\square)$. * $p<0.0001$ between adult and all other rat ages for 25-100 $\mu \mathrm{M}$ GCDC by ANOVA analysis. Results were from three separate experiments. (C) Transmission electron microscopy of mitochondria isolated from rats of different ages (days) at end of MPT experiment. Bar in 36 days panel exposed to GCDC represents $1.0 \mu \mathrm{m}$.

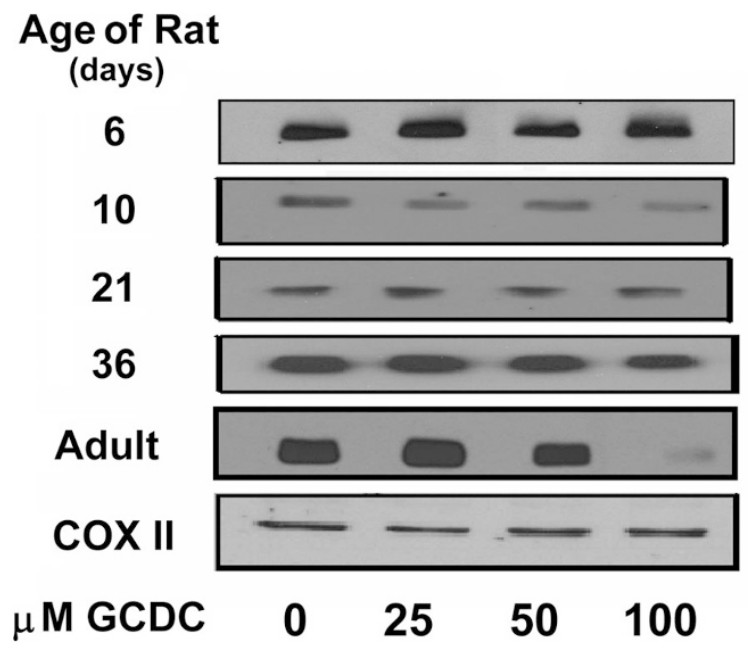

Figure 2. GCDC-induced cytochrome $c$ release from mitochondria. Liver mitochondria from all rat ages were exposed to $0-100 \mu \mathrm{M}$ GCDC during the MPT assay and analyzed for mitochondrial cytochrome $c$ content by immunoblot analysis as described in Supplemental Materials, online. COX II represents subunit of cytochrome $c$ oxidase, which shows equal loading of all lanes. Shown is a representative immunoblot obtained from three independent experiments.

GSH-dependent enzymes were capable of reducing excessive peroxide generation. Among the Bcl-2 family proteins examined, levels of Bak and Bcl-2 were similar between adult and young rat mitochondria (Fig. 4C). In contrast, expression of $\mathrm{Bcl}-\mathrm{xL}$ was higher in mitochondria from the adult rat, whereas 10-day-old rat mitochondria had greater Bax content. These alterations in Bcl-2 proteins would have the combined effect to increase sensitivity, rather than the observed decrease of sensitivity, to MPT induction and cytochrome $c$ release in the 10-day-old rat. Mitochondrial levels of adenine nucleotide translocase, voltage-dependent anion channel, and cyclophilin $\mathrm{D}$ were similar between the 10-day-old and adult rat liver, as were the antioxidant enzymes manganese-superoxide dismutase, thioredoxin (Trx), or thioredoxin reductase (Trx-R) (Fig. $1 S$, supplementary material online at www.pedresearch.org). Thus, neither differences in $\mathrm{Bcl}-2$ proteins and MPT pore proteins, nor antioxidant defenses explained the MPT resistance of young rat mitochondria.

Relationship between MPT induction and $\alpha$-TH levels. Significantly increased $\alpha$-TH concentrations were present in liver homogenate (Fig. 5A) and hepatic mitochondria (Fig. 5B)

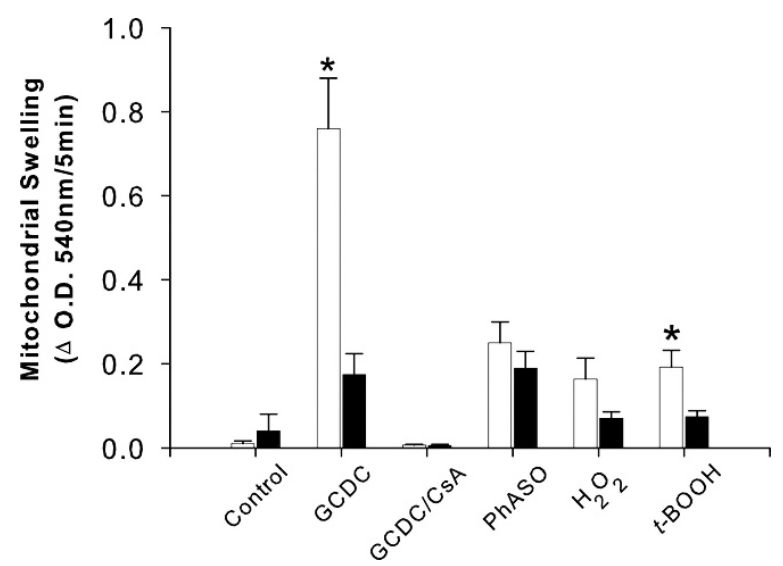

Figure 3. Effect of MPT inducers on swelling of liver mitochondria isolated from adult $(\square)$ and 10-day-old $(\square)$ rats. MPT was induced by incubation with $100 \mu \mathrm{M}$ GCDC, $100 \mu \mathrm{M}$ PhASO, $10 \mathrm{mM} \mathrm{H}_{2} \mathrm{O}_{2}$ or $100 \mu \mathrm{M} t$-BOOH. CsA (5 $\mu \mathrm{M}$ ) was preincubated for 5 min before the addition of $100 \mu \mathrm{M}$ GCDC. ${ }^{*} p<$ 0.01 between adult and 10-day-old art mitochondria using a student $t$ test analysis. Results were from at least five separate experiments.

from 10-day-old rats was compared with adult rats. Neither $\gamma$-TH nor cholesterol differed between adult and young rat mitochondria (data not shown). Ontogenic differences were also observed in expression of $\alpha$-TH transfer protein ( $\alpha$-TTP), a primary regulator of liver $\alpha$-TH secretion, with liver homogenates from the 10-days old containing 63\% less $\alpha$-TTP compared with the adult (Fig. 5C).

To determine whether $\alpha$-TH content was responsible for the age-related differences in MPT susceptibility, we administered s.c. $\alpha$-TH to achieve in vivo mitochondrial $\alpha$-TH levels in adult rats in the range observed in the young rat. Administration of a single s.c. injection of Vital-E 300 resulted in approximately 4-, 7-, and 8-fold increases of mitochondrial $\alpha$-TH concentrations (Fig. 5D), with an inverse correlation observed between MPT induction and mitochondrial $\alpha$-TH concentrations (Fig. 5E). These results suggest that higher mitochondrial $\alpha-\mathrm{TH}$ in young rats may be a factor providing resistance to bile acid-induced MPT.

Cardiolipin levels and relationship to bile acid-induced MPT. Total mitochondrial CL concentrations did not significantly differ between adult and 10-day-old rats (Table 1); however, there were significant differences in individual molecular species of CL between the adult and 10-day-old rats. 
A

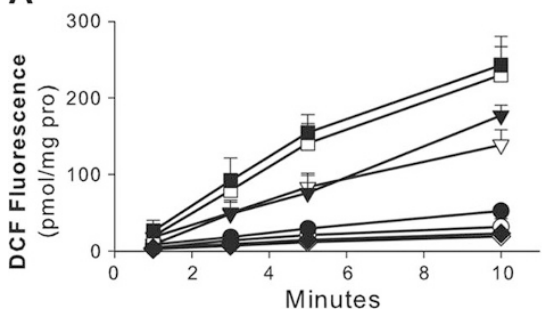

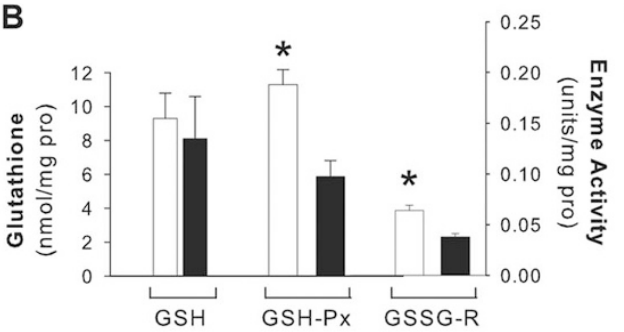

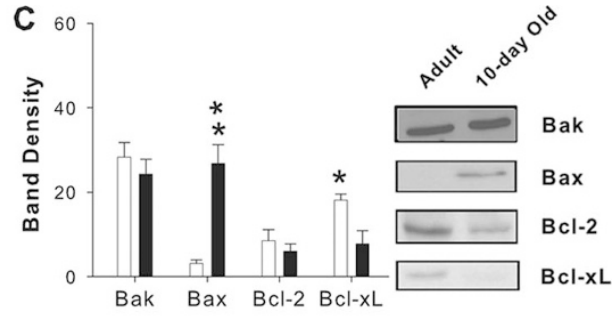

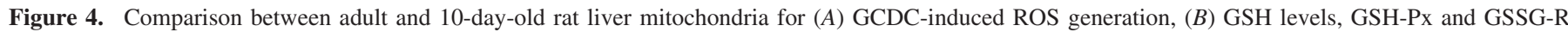

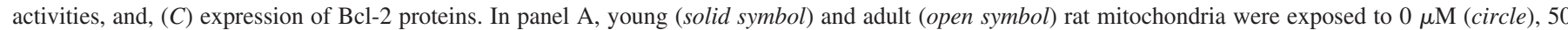

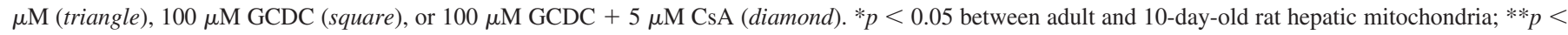
0.005 between adult and 10-days old hepatic mitochondria using a student $t$ test. Results were from at least four separate experiments.

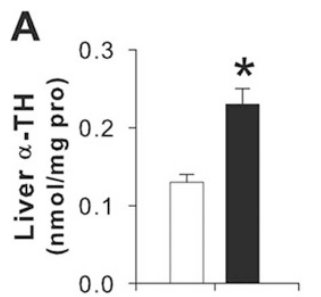

B

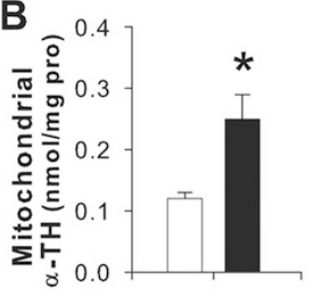

D

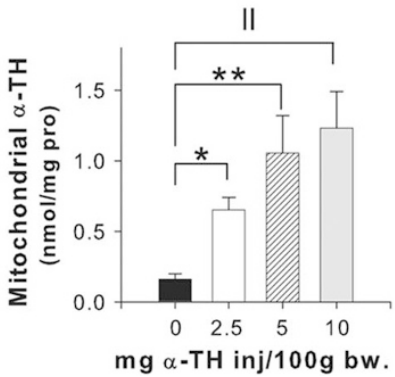

E
C
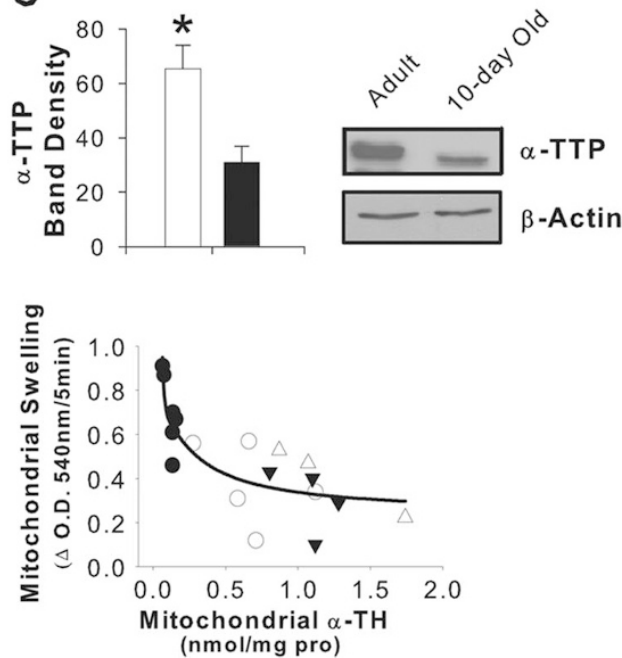

Figure 5. Liver $(A)$ and mitochondrial $(B) \alpha$-TH were significantly higher in 10-day-old rats $(\square)$ compared with adults $(\square)$; * $p<0.05$ between adult and 10 -day-old hepatic mitochondria. (C) $\alpha$-TTP levels by immunoblot in liver homogenate were significantly increased in adult rats compared with the 10-days old. * $p<0.05$ between adult and 10-day-old rat hepatic mitochondria using a student $t$ test. In vivo $\alpha$-TH administration resulted in elevated mitochondrial $\alpha$-TH $(D)$. * $p<0.05$, ** $p<0.005$, $\| p<0.0005$ by ANOVA analysis $(E)$ Rats receiving a single s.c. injection of Vital E-300 (saline- - , 2.5- - , 5- $-\mathbf{\Delta}$, or $10 \mathrm{mg} / 100 \mathrm{~g}$ body weight- $\triangle$ ) exhibited an inverse curvilinear relationship between mitochondrial $\alpha$-TH concentrations and mitochondrial swelling $(r=-0.83, p=0.0007)$ using a regression analysis. Results were from at least three separate experiments (panels $A-C$ ) or at least three animals receiving each SQ dose of $\alpha-\mathrm{TH}$.

The predominant CL species in mammalian mitochondria, TL-CL, was $>1.5$-fold greater in adult mitochondria compared with the 10-days old, and as a percentage of the total CL pool, TL-CL accounted for $52 \%$ and $37 \%$ of the CL present in adult and young rat mitochondria, respectively.

To determine whether young rat MPT resistance was related to their CL concentrations, we increased the CL content of young rat hepatic mitochondria. Mitochondria from young rats incubated with $11.7 \mathrm{nmol} \mathrm{CL} / \mathrm{mL}$ mitochondria did not result in enrichment of either CL or TL-CL (Fig. 6A), or change in GCDC-induced MPT (Fig. 6B). In contrast, significant enrichment occurred with $117 \mathrm{nmol} \mathrm{CL} / \mathrm{mL}$ mitochondria; mitochondria from young rats achieved a 2.9-fold increase in total CL and a 3.9-fold increase in TL-CL concentration resulting in only a modest increase in GCDC-induced mitochondrial swelling (Fig. $6 B$ ). Although a positive correlation was demonstrated between both total CL and TL-CL content in young rat mitochondria (Fig. $6 C$ ), overall these data indicate that elevating CL content in young rat hepatic mitochondria to levels measured in adult mitochondria only slightly increased their susceptibility toward bile acid-induced MPT.

\section{DISCUSSION}

The human neonate is particularly susceptible to cholestatic liver injury by mechanisms not well understood. In this study, we used purified mitochondrial fractions to investigate developmental differences in mitochondrial properties and pathways involved in bile acid-induced cell death. We observed that hepatic mitochondria from young rats were resistant to alterations in permeability induced by bile acids that are retained in cholestasis, and that differences in mitochondrial membrane lipid composition between young and adult rats were related to these findings. It is unclear why purified mitochondria from the young rat, which typically exhibits "physiologic cholestasis" at birth (23), would be resistant to bile acid toxicity. One possible explanation may be, in fact, that this represents a protective response that prevents the normally elevated concentrations of bile acids (related to the immature bile acid secretory pathways that are present in the neonate $(24,25)$ from initiating hepatocellular apoptosis or necrosis during a period of rapid growth of the developing liver. 
Table 1. Total CL and individual CL molecular species in hepatic mitochondria

\begin{tabular}{|c|c|c|c|}
\hline Rat age & CL species (fatty acyl chain) & $\mathrm{nmol} / \mathrm{mg}$ pro $($ mean $\pm \mathrm{SEM})$ & $p$ \\
\hline Adult & $18: 2-18: 2-16: 1-16: 1$ & $0.028 \pm 0.004$ & 0.0001 \\
\hline 10-day old & & $0.069 \pm 0.002$ & \\
\hline Adult & $18: 2-18: 2-16: 1-16: 0$ & $0.032 \pm 0.004$ & 0.002 \\
\hline 10-day old & & $0.074 \pm 0.007$ & \\
\hline Adult & $18: 2-18: 1-16: 1-16: 0$ & $0.021 \pm 0.003$ & 0.019 \\
\hline 10-day old & & $0.033 \pm 0.003$ & \\
\hline Adult & $18: 2-18: 2-18: 2-16: 1$ & $0.240 \pm 0.04$ & 0.038 \\
\hline 10-day old & & $0.143 \pm 0.01$ & \\
\hline Adult & $18: 2-18: 2-18: 2-16: 0$ & $0.152 \pm 0.02$ & 0.47 \\
\hline 10-day old & $18: 2-18: 2-18: 1-16: 1$ & $0.149 \pm 0.01$ & \\
\hline Adult & $18: 2-18: 1-18: 1-16: 1$ & $0.066 \pm 0.009$ & 0.017 \\
\hline 10-day old & $18: 2-18: 2-18: 1-16: 0$ & $0.104 \pm 0.008$ & \\
\hline Adult & $18: 2-18: 2-18: 2-18: 2$ & $3.007 \pm 0.128$ & 0.001 \\
\hline 10-day old & & $1.922 \pm 0.141$ & \\
\hline Adult & $18: 2-18: 2-18: 2-18: 1$ & $1.283 \pm 0.110$ & 0.220 \\
\hline 10-day old & & $1.415 \pm 0.085$ & \\
\hline Adult & $18: 2-18: 2-18: 2-20: 4$ & $0.132 \pm 0.013$ & 0.040 \\
\hline 10-day old & & $0.180 \pm 0.015$ & \\
\hline Adult & $18: 1-18: 2-18: 2-20: 4$ & $0.294 \pm 0.038$ & 0.459 \\
\hline 10-day old & & $0.299 \pm 0.016$ & \\
\hline Adult & $18: 2-18: 2-18: 2-20: 2$ & $0.343 \pm 0.035$ & 0.117 \\
\hline 10-day old & & $0.404 \pm 0.019$ & \\
\hline Adult & $18: 2-18: 2-18: 2-22: 6$ & $0.128 \pm 0.011$ & 0.003 \\
\hline 10-day old & & $0.237 \pm 0.019$ & \\
\hline Adult & $22: 5-18: 2-18: 2-18: 2$ & $0.072 \pm 0.011$ & $<0.0001$ \\
\hline 10-day old & & $0.207 \pm 0.010$ & \\
\hline Adult & Total CL & $5.80 \pm 0.30$ & 0.158 \\
\hline 10-day old & & $5.24 \pm 0.33$ & \\
\hline
\end{tabular}

CL levels were determined by electrospray mass spectrometry as described in Methods. Results were obtained from 4 separate preparations and expressed as nmol Total CL or individual species/mg protein. Acyl group composition of individual species are listed as carbon number:double bonds. Two acyl groups are listed when they could not be separated. Statistically significant differences $(p<0.05)$ in content of individual molecular species of CL between adult and 10-day old rat liver mitochondria are shown in bold.
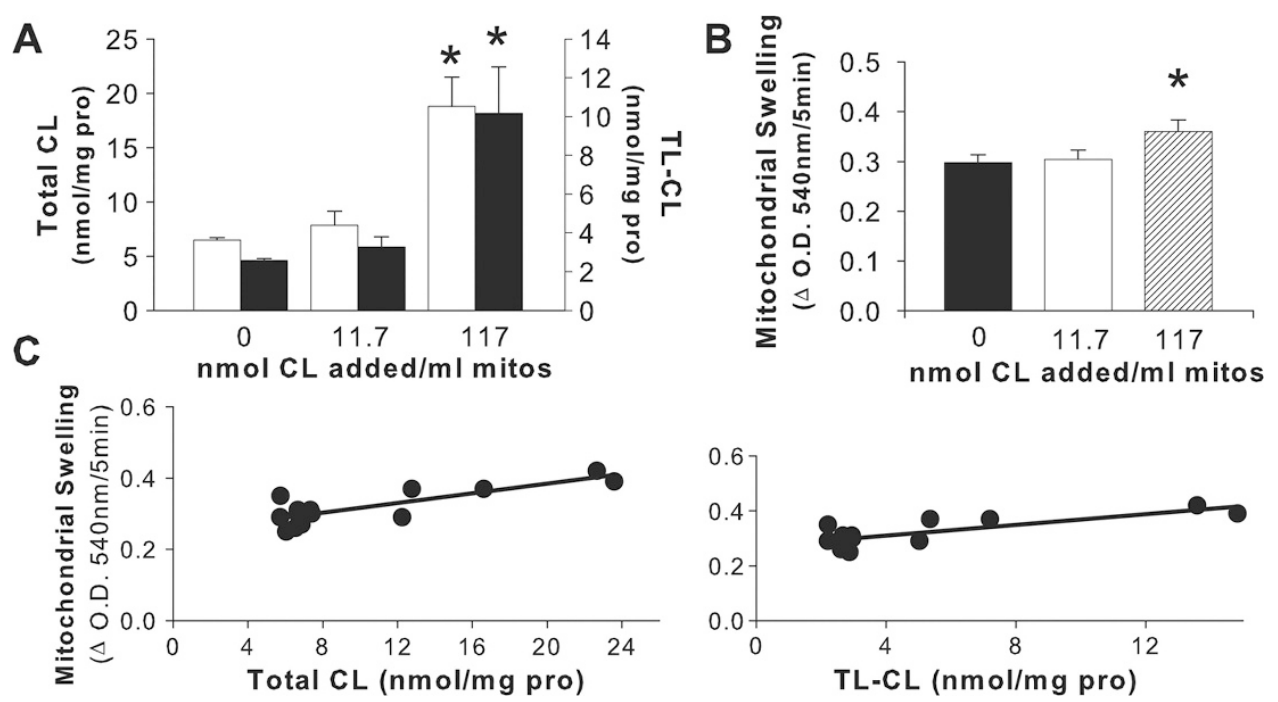

Figure 6. In vitro CL enrichment in mitochondria from young rats resulted in elevated concentrations of total CL ( $\square$ ) and TL-CL ( $\square$ ). (A) Incubation of 117 nmol $\mathrm{CL} / \mathrm{mL}$ mitochondria increased total $\mathrm{CL},(p=0.0002$ for 0 vs $117 \mathrm{nmol} \mathrm{CL} / \mathrm{mL}$ mitochondria) and TL-CL, ( $p=0.0012$ for $0 v s 117 \mathrm{nmol} \mathrm{CL} / \mathrm{mL}$ mitochondria). Incubation of $11.7 \mathrm{nmol} \mathrm{CL} / \mathrm{mL}$ mitochondria failed to significantly increase total CL or TL-CL in mitochondria. (B) Young rat mitochondria incubated with $117 \mathrm{nmol}$ $\mathrm{CL} / \mathrm{mL}$ mitochondria showed a small increase in bile acid-induced MPT compared with control treatment $\left({ }^{*} p \leq 0.05\right)$. Significance was determined by ANOVA analysis. $(C)$ Linear regression analysis revealed a positive relationship between GCDC-induced MPT and total CL concentration $(r=0.82, p<0.001)$ and TL-CL concentration $(r=0.79, p<0.001)$ in hepatic mitochondria of 10-day-old rats. Results were from at least four separate preparations for each CL treatment.

Hydrophobic bile acids directly promote mitochondrial dysfunction or promote cell death by several interrelated pathways, which are regulated by Bcl- 2 family proteins. In the current study, mitochondria from young rats were extremely resistant to bile acid-induced MPT, despite having greater levels of pro-apoptotic Bax and reduced amounts of antiapoptotic Bcl-xL. Thus, the balance of Bcl-2 proteins in young rat liver mitochondria would favor bile acid induction of the 
MPT and cytochrome $c$ release, which was in contrast to the observed MPT resistance in the 10-day-old rat mitochondria (Figs. 1 and 2). Although an earlier study reported no Bcl-2 expression in normal rat hepatocytes (26), we demonstrated the presence of the protein in our purified mitochondria, a finding supported by other groups $(27,28)$. Since Bcl-2 proteins did not explain our MPT findings, we analyzed other factors that could contribute to MPT induction.

Mitochondria from 10-day-old rats contained amounts of adenine nucleotide translocase, voltage-dependent anion channel, and cyclophilin D, putative components of the MPT megapore, similar to adult rat mitochondria. Thus, the structural capability for pore formation was present in the developing rat and MPT resistance was not explained by expression differences in MPT proteins. Inasmuch as bile acid toxicity is associated with significant mitochondrial ROS generation $(29,30)$, it would follow that developmental differences in antioxidant defenses within mitochondria could affect MPT susceptibility. Mitochondria from the 10-day-old rat contained significantly lower GSH-Px and GSSG-R activities, raising the possibility that young rats might not be capable of neutralizing excess hydroperoxides. However, it is unlikely that this reduced activity contributes to their resistance to bile acid-induced MPT, particularly in the presence of adequate GSH concentrations. Furthermore, the young and adult rat mitochondria generated equivalent ROS suggesting that the GSH-dependent enzyme systems functioned similarly in the young and adult rat. Adult rat liver mitochondria contained half of the $\alpha$-TH concentration of young rat liver mitochondria, which corresponded with reduced hepatic expression of $\alpha$-TTP, supporting a previous finding (31). Since $\alpha$-TTP is responsible for transfer of $\alpha$-TH out of the hepatocyte via VLDL secretion (32), lower expression of TTP could be responsible for higher levels of $\alpha-\mathrm{TH}$ in the liver of the young rat, providing increased protection in the developing animal to hepatic-derived oxidative stress. In vivo enrichment of adult rats with s.c. $\alpha$-TH increased resistance to GCDC-induced MPT. This finding supports a possible role of higher mitochondrial $\alpha$-TH concentrations in the protection of the young rat against bile acid toxicity.

Because of its abundance and essential role in mitochondrial function, CL has been investigated in apoptotic cell death $(33,34)$. In one previous study, elevated levels of total CL were associated with reduced liver injury and MPT susceptibility in bile duct-ligated rats (35). Although the current study revealed no difference in total CL between young and adult rat hepatic mitochondria, alterations in the overall profile of individual CL subspecies were observed. In particular, differences in TL-CL concentrations could affect several mitochondrial-dependent processes involved in cell signaling or mitochondrial structure and function. For example, reduced TL-CL in the young rat could alter the ability of CL to bind cytochrome $c$, a high affinity ligand for CL (36); less TL-CL could provide fewer anchor points for hydrophobic and electrostatic binding between CL and cytochrome $c$. The interaction of CL with cytochrome $c$ also promotes a peroxidative activity, which in turn facilitates the release of pro-apoptotic factors from mitochondria $(37,38)$. Finally, a reduction of TL-CL in the young rat may generate less CL hydroperoxides (particularly in the presence of the elevated $\alpha$-TH observed in young rat mitochondria), which have been shown to promote mitochondrial swelling and cytochrome $c$ release from mitochondria (39). In the current study, enrichment of CL and TL-CL into liver mitochondrial membranes of young rats only slightly increased their susceptibility to bile acid-induced MPT, suggesting that MPT resistance in the young rat was not the result of lower basal TL-CL content. It is also possible that other minor CL subspecies could modulate MPT induction through interactions with mitochondrial pore proteins.

In conclusion, we have demonstrated an unexpected resistance of hepatic mitochondria in the young, developing rat to bile acid-induced MPT and cytochrome $c$ release. This phenomenon may be a novel adaptive response that allows the developing hepatocyte to cope with the increased bile acid levels to which it is exposed physiologically, providing protection against the MPT and release of cytochrome $c$, which induce hepatocyte death in the adult rat. Membrane compositional differences between adult and developing young rats are related to this MPT resistance. Further elucidation of the factors responsible for this MPT resistance could potentially be exploited as candidate targets for treatment of cholestasis in older patients, considering the important role of mitochondrial perturbations in the pathogenesis of cholestatic liver injury. Whether similar resistance to MPT is developmentally regulated in other organ systems awaits investigation.

\section{REFERENCES}

1. Arrese M, Ananthananarayanan M, Suchy FJ 1998 Hepatobiliary transport: molecular mechanisms of development and cholestasis. Pediatr Res 44:141-147

2. Phillips MJ 1994 Mechanisms and morphology of cholestasis. In: Suchy FJ (ed) Liver Disease in Children. St. Louis, MO: Moseby, pp 129-144

3. Pittschieler K, Lebenthal E, Bujanover Y, Petell JK 1991 Levels of Cu-Zn and Mn superoxide dismutases in rat liver during development. Gastroenterology 100:10621068

4. Bohles H 1997 Antioxidative vitamins in prematurely and maturely born infants. Int J Vitam Nutr Res 67:321-328

5. Rolo AP, Oliveira PJ, Moreno AJ, Palmeira CM 2000 Bile acids affect liver mitochondrial bioenergetics: possible relevance for cholestasis therapy. Toxicol Sci 57:177-185

6. Krahenbuhl S, Talos C, Sven F, Reichen J 1994 Toxicity of bile acids on the electron transport chain in isolated rat liver mitochondria. Hepatology 19:471-479

7. Krahenbuhl S, Talos C, Reichen J 1994 Mechanisms of impaired hepatic fatty acid metabolism in rats with long-term bile duct ligation. Hepatology 19:1272-1281

8. Palmeira CM, Rolo AP 2004 Mitochondrially-mediated toxicity of bile acids. Toxicology 203:1-15

9. Sokol RJ, Straka MS, Dahl R, Devereaux MW, Yerushalmi B, Gumpricht E, Elkins N, Everson G 2001 Role of oxidant stress in the permeability transition induced in rat hepatic mitochondria by hydrophobic bile acids. Pediatr Res 49:519-531

10. Rodrigues CM, Fan G, Wong PY, Kren BT, Steer CJ 1998 Ursodeoxycholic acid may inhibit deoxycholic acid-induced apoptosis by modulating mitochondrial transmembrane potential and reactive oxygen species production. Mol Med 4:165-178

11. Rodrigues CM, Ma X, Linehan-Stieers C, Fan G, Kren BT, Steer CJ 1999 Ursodeoxycholic acid prevents cytochrome c release in apoptosis by inhibiting mitochondrial membrane depolarization and channel formation. Cell Death Differ 6:842-854

12. Sodeman T, Bronk SF, Roberts PJ, Miyoshi H, Gores GJ 2000 Bile salts mediate hepatocytes apoptosis by increasing cell surface trafficking of fas. Am J Physiol Gastrointest Liver Physiol 278:G992-G999

13. Miyoshi H, Rust C, Roberts PJ, Burgart LJ, Gores GJ 1999 Hepatocyte apoptosis after bile duct ligation in the mouse involves Fas. Gastroenterology 117:669-677

14. Cheng X, Buckley D, Klaassen CD 2007 Regulation of hepatic bile acid transporters Ntcp and Bsep expression. Biochem Pharmacol 74:1665-1676

15. Lawrence RA, Burk RF 1976 Glutathione peroxidase activity in selenium-deficient rat liver. Biochem Biophys Res Commun 71:952-958

16. Carlberg I, Mannervik B 1985 Glutathione reductase. Methods Enzymol 113:484490

17. Lang JK, Gohil K, Packer L 1986 Simultaneous determination of tocopherols, ubiquinols, and ubiquinones in blood, plasma, tissue homogenates, and subcellular fractions. Anal Biochem 157:106-116 
18. Podda M, Weber C, Traber MG, Packer L 1996 Simultaneous determination of tissue tocopherols, tocotrienols, ubiquinols, and ubiquinones. J Lipid Res 37:893-901

19. Gumpricht E, Dahl R, Devereaux MW, Sokol RJ 2005 Licorice compounds glycyrrhizin and 18beta-glycyrrhetinic acid are potent modulators of bile acid-induced cytotoxicity in rat hepatocytes. J Biol Chem 280:10556-10563

20. Gumpricht E, Devereaux MW, Traber M, Sokol RJ 2004 Enrichment of rat hepatic organelles by vitamin E administered subcutaneously. Free Radic Biol Med 37:1712-1717

21. Sparagna GC, Johnson CA, McCune SA, Moore RL, Murphy RC 2005 Quantitation of cardiolipin molecular species in spontaneously hypertensive heart failure rats using electrospray ionization mass spectrometry. J Lipid Res 46:1196-1204

22. Hackenbrock CR, Chazotte B 1986 Lipid enrichment and fusion of mitochondrial inner membranes. Methods Enzymol 125:35-45

23. Belknap WM, Balistreri WF, Suchy FJ, Miller PC 1981 Physiologic cholestasis II: serum bile acid levels reflect the development of the enterohepatic circulation in rats. Hepatology 1:613-616

24. Rippin SJ, Hagenbuch B, Meier PJ, Stieger B 2001 Cholestatic expression pattern of sinusoidal and canalicular organic anion transport systems in primary cultured rat hepatocytes. Hepatology 33:776-782

25. Gao B, St Pierre MV, Stieger B, Meier PJ 2004 Differential expression of bile salt and organic anion transporters in developing rat liver. J Hepatol 41:201-208

26. Kurosawa H, Que FG, Roberts LR, Fesmier PJ, Gores GJ 1997 Hepatocytes in the bile duct-ligated rat express Bcl-2. Am J Physiol 272:G1587-G1593

27. Janiak F, Leber B, Andrews DW 1994 Assembly of Bcl-2 into microsomal and outer mitochondrial membranes. J Biol Chem 269:9842-9849

28. Motoyama S, Kitamura M, Saito S, Minamiya Y, Suzuki H, Saito R, Terada K, Ogawa J, Inaba H $1998 \mathrm{Bcl}-2$ is located predominantly in the inner membrane and crista of mitochondria in rat liver. Biochem Biophys Res Commun 249:628-636

29. Yerushalmi B, Dahl R, Devereaux MW, Gumpricht E, Sokol RJ 2001 Bile acidinduced rat hepatocyte apoptosis is inhibited by antioxidants and blockers of the mitochondrial permeability transition. Hepatology 33:616-626

30. Sokol RJ, Dahl R, Devereaux MW, Yerushalmi B, Kobak GE, Gumpricht E 2005 Human hepatic mitochondria generate reactive oxygen species and undergo the permeability transition in response to hydrophobic bile acids. J Pediatr Gastroenterol Nutr 41:235-243

31. Kim HS, Arai H, Arita M, Sato Y, Ogihara T, Tamai H, Inoue K, Mino M 1996 Age-related changes of alpha-tocopherol transfer protein expression in rat liver. J Nutr Sci Vitaminol (Tokyo) 42:11-18

32. Traber MG, Burton GW, Hamilton RL 2004 Vitamin E trafficking. Ann N Y Acad Sci 1031:1-12

33. Chicco AJ, Sparagna GC 2007 Role of cardiolipin alterations in mitochondrial dysfunction and disease. Am J Physiol Cell Physiol 292:C33-C44

34. Matsko CM, Hunter OC, Rabinowich H, Lotze MT, Amoscato AA 2001 Mitochondrial lipid alterations during Fas- and radiation-induced apoptosis. Biochem Biophys Res Commun 287:1112-1120

35. Lieser MJ, Park J, Natori S, Jones BA, Bronk SF, Gores GJ 1998 Cholestasis confers resistance to the rat liver mitochondrial permeability transition. Gastroenterology 115:693-701

36. Basova LV, Kurnikov IV, Wang L, Ritov VB, Belikova NA, Vlasova II, Pacheco AA, Winnica DE, Peterson J, Bayir H, Waldeck DH, Kagan VE 2007 Cardiolipin switch in mitochondria: shutting off the reduction of cytochrome $\mathrm{c}$ and turning on the peroxidase activity. Biochemistry 46:3423-3434

37. Bayir H, Fadeel B, Palladino MJ, Witasp E, Kurnikov IV, Tyurina YY, Tyurin VA, Amoscato AA, Jiang J, Kochanek PM, DeKosky ST, Greenberger JS, Ahvedova AA, Kagan VE 2006 Apoptotic interactions of cytochrome c: redox flirting with anionic phospholipids within and outside of mitochondria. Biochim Biophys Acta 1757:648-659

38. Kagan VE, Tyurin VA, Jiang J, Tyurina YY, Ritov VB, Amoscato AA, Osipov AN, Belikova NA, Kapralov AA, Kini V, Vlasova II, Zhao Q, Zou M, Di P, Svistunenko DA, Kurnikov IV, Borisenko GG 2005 Cytochrome c acts as a cardiolipin oxygenase required for release of proapoptotic factors. Nat Chem Biol 1:223-232

39. Petrosillo G, Casanova G, Matera M, Ruggiero FM, Paradies G 2006 Interaction of peroxidized cardiolipin with rat-heart mitochondrial membranes: induction of permeability transition and cytochrome c release. FEBS Lett 580:6311-6316 